\title{
THE RING $C(X, R)$ CONSIDERED AS A SUBRING OF THE RING OF ALL REAL-VALUED FUNCTIONS
}

LYLE E. PURSELL

Conditions under which the ring $C(X, R)$ of all real-valued continuous functions on a completely regular space $X$ determines $X$ have been investigated in a number of papers [1-5]. Gelfand and Kolmogoroff [2] have shown that if $X$ is a compact completely regular space, then the space of maximal ideals in $C(X, R)$ with the Stone topology is homeomorphic to $X$ and hence $C(X, R)$ determines $X$. The most general result for noncompact spaces has been obtained by F. W. Anderson [1] who has shown that the lattice of all real-valued continuous functions on a topological space determines this space if it is a completely regular space all of whose points are $G$-delta sets. Since $f(x) \geqq g(x)$ for all $x$ if and only if $f-g$ has square root in $C(X, R)$, a ring isomorphism of $C(X, R)$ implies a lattice isomorphism and Anderson's result is equivalent to the statement that the ring $C(X, R)$ determines $X$ if $X$ is a completely regular space all of whose points are $G$-delta sets.

In this paper we show that if $X$ is completely regular, then $X$ is topologically determined by the pair of rings consisting of the ring $R^{X}$ of all real-valued functions on $X$ and its subring $C(X, R)$.

Lemma 1. An ideal I in $C(X, R)$ is fixed if and only if there exists a function $g$ in $R^{X}$ such that $0 \leqq g(x) \leqq 1$ for all $x$ in $X, g\left(x_{0}\right)=0$ for some $x_{0}$ and if $f$ is in $I$ and $-1 \leqq f(x) \leqq 1$ for all $x$, then $-g(x) \leqq f(x) \leqq g(x)$ for all $x$.

Proof. If all functions in $I$ vanish at some point $x_{0}$, then we observe that if we define $g(x)=1$ for $x \neq x_{0}$ and $g\left(x_{0}\right)=0$, then $g$ is the desired function.

Conversely, assume that for an ideal $I$ such a function $g$ exists. If $f$ is continuous, then the function $h$ defined by $h(x)=f(x)$ if $-1 \leqq f(x)$ $\leqq 1$ and $h(x)=1 / f(x)$ otherwise is continuous. If $f$ is in $I$, then $f h$ is in $I,-1 \leqq(f h)(x) \leqq 1$ for all $x$, and $(f h)(x)=0$ if and only if $f(x)=0$. Hence $-g(x) \leqq(f h)(x) \leqq g(x)$ for all $x,(f h)\left(x_{0}\right)=0$, and, accordingly, $f\left(x_{0}\right)=0$. Therefore $I$ is a fixed ideal.

Since every automorphism of $R^{x}$ preserves the relation $f(x) \leqq g(x)$

Presented to the Society, November 23, 1956 under the title The ring of all realvalued continuous functions considered as a subring of the ring of all real-valued functions; received by the editors November 13, 1956. 
for all $x$, then every automorphism of $R^{X}$ which maps $C(X, R)$ onto itself will map a fixed ideal of $C(X, R)$ onto a fixed ideal of $C(X, R)$.

THEOREM 1. If $X$ is a completely regular topological space, then the space of fixed maximal ideals with the Stone topology (i.e. a collection of fixed maximal ideals is closed if and only if their intersection $J$ is equal to the intersection of all fixed maximal ideals which contain $J$ ) is homeomorphic to $X$.

The proof is similar to the proof [2] that the set of maximal ideals in $C(X, R)$ with the Stone topology is homeomorphic to $X$ if $X$ is compact. One observes that if $C(X, R)$ is a separating class for $X$ (i.e. if $x_{1} \neq x_{2}$, then there is a function $f$ in $C(X, R)$ such that $f\left(x_{1}\right)$ $\left.\neq f\left(x_{2}\right)\right)$ but $X$ is not completely regular, then there is a one-to-one correspondence between $X$ and the space of fixed maximal ideals but the latter space will have fewer closed sets.

From the above results we obtain:

THEOREM 2. If $X$ and $Y$ are two completely regular spaces and if $R^{X}$ and $R^{Y}$ are isomorphic under an isomorphism which maps $C(X, R)$ onto $C(Y, R)$, then $X$ and $Y$ are homeomorphic.

Lemma 1 and Theorems 1 and 2 also hold if $C(X, R)$ is replaced by the ring $C^{*}(X, R)$ of all bounded continuous functions on $X$.

\section{BIBLIOGRAPHY}

1. F. W. Anderson, $A$ lattice characterization of completely regular G-delta spaces, Proc. Amer. Math. Soc. vol. 6 (1955) pp. 757-765.

2. I. Gelfand and A. N. Kolmogoroff, On rings of continuous functions on topological spaces, C. R. (Doklady) Acad. Sci. URSS. vol. 22 (1939) pp. 11-15.

3. L. Gillman, M. Henriksen and M. Jerison, On a theorem of Gelfand and Kolmogoroff concerning maximal ideals in rings of continuous functions, Proc. Amer. Math. Soc. vol. 5 (1954) pp. 447-455.

4. E. Hewitt, Rings of real-valued continuous functions. I, Trans. Amer. Math. Soc. vol. 64 (1948) pp. 45-99.

5. L. E. Pursell, An algebraic characterization of fixed ideals in certain function rings, Pacific Journal of Mathematics vol. 5 (1955) pp. 963-969.

Grinnell College 